\title{
Morte e Morrer na emergência pediátrica: a protagonização da equipe de enfermagem frente a finitude da vida
}

\author{
Death and dying in the pediatric emergency: the role of the nursing team facing the finitude of life \\ Muerte y morir en la emergencia pediátrica: el papel del equipo de enfermería frente a la finitud de \\ la vida
}

\author{
Wanderson Alves Ribeiro', Bruna Porath Azevedo Fassarella², Keila do Carmo Neves ${ }^{3}$
}

Como citar esse artigo. Ribeiro, WA; Fassarella, BPA; Neves, KC. Morte e Morrer na emergência pediátrica: a protagonização da equipe de enfermagem frente a finitude da vida. Revista Pró-UniverSUS. 2020 Jan./Jun.; 11 (1): 123-128.

\begin{abstract}
Resumo
Trata-se de uma pesquisa bibliográfica de abordagem qualitativa e caráter descritivo que tem como objeto de estudo o processo morte e morrer na emergência pediátrica, que tem como objetivos descrever o processo de finitude da vida no setor da emergência pediátrica e identificar a percepção e atuação da equipe de enfermagem na finitude da vida no setor de emergência pediátrica. O findar da vida na infância é considerado mais doloroso que na vida adulta, já que a morte de uma criança leva a um conceito de tragédia e interrupção do ciclo da vida. Dessa maneira, a sobrevida dela se constitui como um objetivo principal para equipe, devido à alta possibilidade de cura em pacientes pediátricos. Como metodologia, utilizou-se a Biblioteca Virtual de Saúde, nas bases de informações LILACS, BDENF, MEDLINE e SCIELO, com recorte temporal de 2009 à 2019.Conclui-se que o enfrentamento do processo morte e morrer emerge diversas complicações pra equipe de enfermagem e, quando ocorre na idade pediátrica, em situações de emergência é ainda mais desfavorável e, por isso, precisa ser tratado com muito cuidado e cautela. Palavras-chave: Enfermagem, Enfermagem Pediátrica, Morte.
\end{abstract}

\begin{abstract}
This is a qualitative and descriptive bibliographical research that has as its object of study the process of death and dying in the pediatric emergency, which aims to describe the process of finitude of life in the pediatric emergency sector and to identify the perception and performance.of nursing staff on the finitude of life in the pediatric emergency department. The ending of life in childhood is considered more painful than in adulthood, as the death of a child leads to a concept of tragedy and disruption of the life cycle. Thus, its survival is a major goal for the team, due to the high possibility of cure in pediatric patients. As methodology, we used the Virtual Health Library, in the databases LILACS, BDENF, MEDLINE and SCIELO, with timeframe from 2009 to 2019. It is concluded that facing the death and dying process emerges several complications for the nursing team. and when it occurs at pediatric age, in emergency situations it is even more unfavorable and therefore needs to be treated with great care and caution.

Keywords: Nursing, Pediatric nursing, Death.
\end{abstract}

\section{Resumen}

Esta es una investigación bibliográfica cualitativa y descriptiva que tiene como objeto de estudio el proceso de muerte y muerte en la emergencia pediátrica, cuyo objetivo es describir el proceso de finitud de la vida en el sector de emergencia pediátrica e identificar la percepción y el rendimiento. Del personal de enfermería sobre la finitud de la vida en el departamento de emergencias pediátricas. El final de la vida en la infancia se considera más doloroso que en la edad adulta, ya que la muerte de un niño conduce a un concepto de tragedia e interrupción del ciclo vital. Por lo tanto, su supervivencia es un objetivo importante para el equipo, debido a la alta posibilidad de curación en pacientes pediátricos. Como metodología, utilizamos la Biblioteca Virtual de Salud, en las bases de datos LILACS, BDENF, MEDLINE y SCIELO, con un calendario de 2009 a 2019. Se concluye que enfrentar el proceso de muerte y muerte surge varias complicaciones para el equipo de enfermería. y cuando ocurre en la edad pediátrica, en situaciones de emergencia es aún más desfavorable y, por lo tanto, debe tratarse con mucho cuidado y precaución.

Palabras clave: Enfermería, Enfermería pediátrica, Muerte

Afiliação dos autores: 1. Enfermeiro. Mestre pelo Programa Acadêmico em Ciências do Cuidado em Saúde pela Escola de Enfermagem Aurora de Afonso Costa da UFF, Niterói/ RJ. Pós-Graduando em Alta Complexidade com ênfase em CTI (UNIGRANRIO0; Saúde da Família (UNIRIO); Informática em Saúde (UNIFESP); Nefrologia Multidisciplinar (UFMA); Pediatria e Neonatologia (FAVENI); Enfermagem em Oncologia (IBRA); Gestão de Redes de Atenção à Saúde (FIOCRUZ); Enfermagem em Estomaterapia (UERJ). Professor Substituto no Departamento de Enfermagem Fundamental da Escola de Enfermagem Anna Nery/UFRJ. Docente do Curso de Graduação da UNIG e UCB

2. Enfermeira. Mestranda em Ciências Aplicadas em Saúde da Universidade Severino Sombra. Docente do Curso de Graduação da UNIG. Preceptora Acadêmica do Curso de Graduação em Enfermagem da UNIABEU.

3. Enfermeira. Pós-Graduada em Nefrologia; Mestre e Doutora em Enfermagem pela UFRJ. Docente do Curso de Graduação em Enfermagem da UNIG.

* Email de correspondencia: keila_arcanjo@hotmail.com 


\section{Introdução}

Afinitude da vida é o momento em que não existem mais possibilidades de resgatar as condições de saúde de um indivíduo, sendo a morte, inevitável e previsível. O seu caminho o direciona para a morte iminente, sem chances de reestabelecer o seu caminho para gozar de plena saúde. Diante a terminalidade da vida, a equipe de enfermagem precisa oferecer todos os cuidados paliativos disponíveis sem que estes tenham o objetivo de aplicação de diagnósticos, e terapêuticas inúteis, respeitando a decisão do paciente ou do representante legal, em casos de impossibilidade do mesmo. ${ }^{1,2}$

O findar da vida na infância é considerado mais doloroso que na vida adulta, já que a morte de uma criança leva a um conceito de tragédia e interrupção do ciclo da vida. Dessa maneira, a sobrevida dela se constitui como um objetivo principal para equipe, devido à alta possibilidade de cura em pacientes pediátricos. ${ }^{3}$

Diante as internações hospitalares, o setor de emergência pediátrica compreende uma taxa alta de internações, um grande número de crianças é acometido por doença graves e avançadas com prognostico ruim, sendo a morte um destino inevitável para muitas delas. Muitas destas doenças causam sequelas graves que reduzem a expectativa de vida, que aumentam a frequência das internações hospitalares. ${ }^{4,5}$

A morte faz parte do desenvolvimento natural do ser humano, todos estão destinados a morrer em algum instante, sejam por causas naturais, doenças, acidentes e outros. Lidar com esta situação é uma realidade vivenciada por muitas pessoas, mas neste estudo a ênfase será no profissional da área da saúde. ${ }^{4}$

Uma criança reflete para qualquer indivíduo o início da vida, as possiblidades de crescimento e desenvolvimento saudáveis. Lidar com a morte de uma criança traz sentimentos dolorosos para familiares e profissionais de saúde, é complexo entender a descontinuidade do processo natural da vida. É processo muito mais doloroso para as pessoas, pelo processo prematuro da finitude da vida. ${ }^{6}$

Esta realidade de finitude da vida ocorre em diferentes setores da área hospitalar, mas neste estudo o foco estará no setor de pediatria, e assim como qualquer paciente adulto, uma criança sofre com o processo de adoecimento e também precisa lidar com o fim da vida. $\mathrm{O}$ que se tem observado é que a equipe de enfermagem não está conseguindo vivenciar com a finitudeda vida neste contexto de trabalho e acabam tendo consequências físicas e psicológicas diante a morte das crianças. ${ }^{6}$

Assim como a maioria das pessoas, a equipe de enfermagem se mostram assustados ou com medo de abordar sobre o assunto, a justificativa usada por muitos deles, é que foram preparados para salvar vidas e garantir a manutenção dela, e ao estarem diante da morte preferem não aceitar, negam que o fenômeno pode acontecer. ${ }^{7}$

O processo da morte e do morrer precisa ser inserido na realidade da equipe de enfermagem, preparando ele para o acontecimento, suporte ao paciente durante o processo, o suporte aos familiares, e também sem deixar que isto venha comprometer sua vida e sua saúde. Este desafio precisa ser vencido pelos profissionais da saúde, e principalmente pelos da enfermagem, que passam todo o tempo de internação junto ao paciente.

A dor da perda em relação a uma criança é muito maior quando comparada a um idoso. Quando uma morte pediátrica acontece sempre surge o questionamento: por que aconteceu com a criança que ainda tinha toda uma vida pela frente? Esse questionamento tende a despertar os sentimentos de negação, de impotência, de perda, de tristeza nos profissionais de enfermagem e em todos os familiares. $^{3}$

$\mathrm{O}$ processo de morte e morrer na pediatria, especialmente, costuma resultar em impacto maior. Confrontar a morte por si só é uma questão difícil, mas a sua ocorrência em uma fase precoce, ou seja, nos primeiros anos de vida de uma pessoa, implica dificuldades de compreensão e aceitação. Estudos comprovam que os enfermeiros de UTI pediátrica sofrem mais com o processo de morte, pois entendem que a vida da criança foi interrompida e que fracassou por não conseguir evitar a ocorrência do óbito. ${ }^{3}$

Diante da problemática apresentada pode-se destacar como objeto de estudo o processo Morte e Morrer na emergência pediátrica.

Para tal, traçou-se a seguinte questão norteadora: Qual a percepção da equipe de enfermagem na finitude da vida no setor de emergência pediátrica?

Diante disso, a pesquisa tem como objetivo descrever o processo de finitude da vida no setor da emergência pediátrica; identificar a percepção e atuação da equipe de enfermagem na finitude da vida no setor de emergência pediátrica.

\section{Metodologia}

Entende-se por metodologia todas as ciências caracterizam-se pela utilização de métodos científicos; em contrapartida, nem todos os ramos de estudo que empregam estes métodos são ciências. Dessas afirmações podemos concluir que a utilização de métodos científicos não é da alçada exclusiva da ciência, mas não há ciência sem o emprego de métodos científicos. ${ }^{8}$

Trata-se de uma pesquisa bibliográfica de abordagem qualitativa e caráter descritivo. Cabe ressaltar que a pesquisa bibliográfica que é desenvolvida com auxílio de material já elaborado, constituído principalmente de livros e artigos científicos. Contudo 
em grande parte dos estudos seja exigido algum tipo de trabalho deste gênero, há pesquisas desenvolvidas exclusivamente a partir de fontes bibliográficas. ${ }^{9}$

Em relação ao método qualitativo, discorre que é o processo aplicado ao estudo da biografia, das representações e classificações que os seres humanos fazem a respeito de como vivem, edificam seus componentes e a si mesmos, sentem e pensam. ${ }^{10}$

De acordo as pesquisas descritivas possuem como objetivo a descrição das características de uma população, fenômeno ou de uma experiência. ${ }^{10}$

Os dados foram coletados em base de dados virtuais. Para tal utilizou-se a Biblioteca Virtual de Saúde(BVS), na seguinte base de informação: Literatura Latino-Americana e do Caribe em Ciências da Saúde (LILACS), Literatura Internacional em Ciência da Saúde (MEDLINE), Scientific Eletronic Library Online (SCIELO), dentre outros, no período de Abril à Junho de 2019.

Optou-se pelos seguintes descritores: Enfermagem; Enfermagem Pediátrica e Morte que se encontram nos Descritores em Ciência da Saúde (DECS).

Estabeleceu-se então para a realização da pesquisa os critérios de inclusão: textos na íntegra, artigos científicos e em português com abordagem da temática estabelecida e que obedecessem ao recorte temporal de 2009 a 2019 e como critérios de exclusão, os textos incompletos, dissertações, teses e em língua estrangeira, textos que não abordassem a temática estabelecida e com recorte temporal inferior a 2009.

Cabe mencionar que os textos em língua estrangeira foram excluídos devido o interesse em embasar o estudo com dados do panorama brasileiro e os textos incompletos, para oferecer melhor compreensão através da leitura de textos na integra.

Após a associação dos descritores em tríades foram encontrados 398 artigos, excluídos 387 e selecionados apenas 11 .

Com vista a ampliar o conhecimento, a recorrência e o estado da temática, foi realizada uma pesquisa bibliográfica no endereço eletrônico scholar. google.com.br, para embasamento e contextualização do tema em questão, onde foram aplicados os critérios de inclusão e exclusão e selecionados 02 artigos.

\section{Analise de dados e discussão de resultados}

Subsequente a seleção dos artigos, foi realizado uma leitura reflexiva dos trezes artigos, emergiu duas categorias: O processo de finitude da vida no setor da emergência pediátrica e a percepção, atuação e impacto da equipe de enfermagem na finitude da vida no setor de emergência pediátrica.

\section{$1^{\text {a }}$ Categoria: Aspectos conceituais e o processo de finitude da vida no setor da emergência pediátrica}

No que se refere a abordar sobre a finitude da vida, é necessário também compreender sobre o conceito da morte. Em que um dos significados é o fim da vida, destruição e ruína. Existe um tabu em relação à morte, os costumes e culturas traçados pela sociedade direcionam o indivíduo a valorização da vida e pouco se introduz o assunto ao longo da construção de conhecimento das pessoas. ${ }^{11,12}$

Historicamente, lidar com a morte ou a finitude da vida traz diferentes vertentes de estudo. A partir do Renascimento, os indivíduos começaram a transformar sua mentalidade, com o objetivo de ampliar o saber. E a morte neste período não era aceita, pois simplesmente não era conhecida e difundida, apenas percebia-se a morte como um desaparecimento da individualidade. Os mortos eram colocados nas sepulturas, mas a sociedade aceitava que eles ainda estariam convivendo com os vivos. ${ }^{11}$

Na Idade Média, passou a ser vista como uma fase necessária para toda pessoa, uma condição que todos alcançariam. Com os avanços tecnológicos e transformações da sociedade, o capitalismo foi escolhido como modelo econômico estruturante da sociedade e junto a ele a desvalorização do entendimento sobre a morte. O importante seria desenvolver a intelecto da pessoa para o progresso e acumulação de riquezas, tudo voltada apenas para o viver. ${ }^{11}$

Com o tempo a morte passou a ser vista como uma condição inevitável para a pessoa que chegou a velhice e construiu-se na sociedade que a morte de uma pessoa jovem não é aceitável. Relacionando a morte em idades avançadas como um processo natural e em pessoas jovens, uma causalidade que leva a angústia e escândalo. E tais pensamentos se difundiram, até que as transformações constantes da sociedade tornaram esta ideia obsoleta. ${ }^{11}$

Recentemente observa-se que idosos tem adquirido novos hábitos de vida, que podem levar ao prolongamento da vida e a redução de adoecimento durante esta fase. E não menos comum, o aumento da quantidade de adoecimento atingindo pessoas cada vez mais jovens, e morte estando presente em qualquer idade. ${ }^{11}$

Para à temática da saúde, o conceito de finitude da vida abrange o esgotamento das possibilidades terapêuticas de retomada das condições de saúde ideais e a inevitável aproximação à morte. A relevância para os profissionais de saúde é a qualidade de vida da pessoa durante o processo até o momento final, e não se deve focar apenas na quantidade de tempo para a morte. ${ }^{13}$

$\mathrm{O}$ indivíduo traça uma trajetória em direção ao 
fim da vida, mas é necessário ser compreendido que não significa não ter mais o que fazer. Pois, estas pessoas ainda necessitam de acompanhamento terapêutico, este com outra finalidade, diferente da cura. ${ }^{14}$

Tais finalidades englobam alívio da dor, diminuição do desconforto e suporte durante todo o processo. Estas precisam ser entendidas, tanto pelo indivíduo, como pela família e profissionais de saúde presentes durante o processo. ${ }^{14}$

Mas é interessante ressaltar que o objetivo dos profissionais da área da saúde concentra-se na ajuda, cuidado, melhora e cura dos vivos, e pouco se desenvolve os estudos sobre a morte, somente em disciplinas específicas, e que foram construídas na comunidade científica para este fim. Por exemplo, a vertente para medicina forense ou legal, mérito de estudo que não será aprofundado nesta pesquisa. ${ }^{13}$

A complexidade de abordagem sobre a morte ou a finalidade da vida está nas inquietações, medos e dúvidas que entornam a conceito e suas características para a sociedade. Para qualquer indivíduo é difícil lidar com a morte, mesmo tendo o conhecimento que esta pode ocorrer a qualquer momento, um fato não evitável. Todos morrerão, o fato da morte acontecerá, o processo de morrer é que será diferenciado para cada pessoa. ${ }^{13}$

E esta abordagem para equipe de enfermagem da emergência pediátrica é entendida como uma barreira no caminho, um quebra do objetivo da profissão. Tais profissionais não são adequadamente preparados durante a graduação para lidar com a morte durante o processo de trabalho. Ela é citada em diferentes momentos, mas somente com a experiência do fato é possível saber o que realmente a morte causará no indivíduo. Pois, cada pessoa terá uma resposta física, psicológica e social para a finalidade da vida. ${ }^{13}$

A atuação da equipe de enfermagem na rede hospitalar abrange também o cuidado a crianças e adolescentes que estão acometidos por doenças crônicas e graves, que podem direcionar aquele indivíduo ao processo de morrer. Nestas situações ocorre a articulação entre a assistência prestada e a morte. Sendo uma atuação complexa e de difícil compreensão para os profissionais que mesmo capacitados, não sabem lidar com a finitude da vida. ${ }^{15}$

Em determinadas internações, pediátricas ou não, o desfecho do paciente poderá ser a morte, fato este que deveria ser esperado pelos que atuam na assistência. No caso da emergência pediátrica, pela cultura da sociedade, vincula-se a criança ao desenvolvimento futuro, uma esperança, o início de uma trajetória, e ter que aceitar que uma criança vai terminar sua vida, antes mesmo de desenvolvê-la é altamente desgastante, traz sofrimento para familiares, amigos e equipe de enfermagem, devido à reação mais intensa e subjetiva das pessoas diante a morte. ${ }^{15}$

\section{$2^{a}$ Categoria: A percepção, atuação e impacto da equipe de enfermagem na finitude da vida no setor de emergência pediátrica}

Muito se discute que os determinantes da vida do ser humano irão influenciar no seu estado de saúde. A morte, fato comum em serviços de atendimento hospitalar e principalmente, urgência e emergência, podem ser consideradas um condicionante do ambiente de trabalho, que interfere diretamente no profissional. ${ }^{15}$

Em maior frequência que as outras especialidades, a equipe de enfermagem tem contato contínuo com o processo de morte de diversos pacientes e de diferentes faixas etárias. Esta equipe assume que o enfrentamento à morte na pediatria é complexo, e poucos são os que não se afetam pelo ocorrido. ${ }^{15}$

Antes de abordar a atuação da enfermagem na finitude da vida na emergência pediátrica, valem ressaltar algumas atividades exercidas pela equipe na emergência pediátrica, as diversas habilidades exigidas por um setor complexo. A equipe de enfermagem, além de serem responsáveis pelo acompanhamento clínico das crianças internadas, realizam diferentes atividades terapêuticas, como diversos curativos, ventilação mecânica, manipulação de drogas vasoativas, cateterismo vesical e outros. ${ }^{16}$

De acordo estudos não existiam intervenções para a finitude da vida, apenas direcionadas ao preparo do corpo, após a morte. Realizando-se pesquisas, foram discutidas abordagens para que fosse possível a equipe de enfermagem atuar no cuidado para a finitude da vida. $^{16}$

Como se encontra na pesquisa científica realizada por outros autores, os profissionais já demonstram e assumem a dificuldade em aceitar que as crianças internadas nestas unidades poderão morrer. Para entender sobre a atuação da enfermagem é preciso ter ciência de que o enfermeiro e sua equipe estarão acompanhando o processo da finitude da vida, período de vida restante, a morte e o cuidado dos familiares após a morte. ${ }^{17}$

Ressalta que a atividade não somente da equipe de enfermagem, mas também de toda equipe multiprofissional é oferecer suporte, informação, conforto e dignidade ao paciente e sua família por meio da assistência. São necessários estes suportes, pois a criança vivencia sentimentos de medo, abandono, culpa e comportamentos depressivos. A enfermagem atua nos setores de pediatria através do cuidado humanizado, as categorias de trabalho abrangem o levantamento das necessidades da criança, da família e institucionais, relações interpessoais, prescrições e intervenções à saúde da criança. ${ }^{17}$

$\mathrm{Na}$ assistência de enfermagem, inclui-se a manipulação dos equipamentos tecnológicos presentes 
nas unidades de assistência para o suporte de vida e mudanças no processo de morrer, um exemplo é o ventilador mecânico. Este fator modificou a visão dos profissionais, além influenciar a prática e os comportamentos adotados diante aos indivíduos que estão em finitude, mesmo que prolongado por estes suportes. ${ }^{18}$

A experiência do enfermeiro na prática assistencial na emergência pediátrica influencia diretamente no desenvolvimento das habilidades, o domínio da assistência vai aumentando com o tempo de atuação. E as ações tornam-se mais conhecidas, mas continuam sendo desafiadoras para profissional, como é o caso das situações de morte e uso de tecnologias. ${ }^{18}$

Muitos enfermeiros novos na profissão ao deparar-se com estes desafios podem ficar em estado de choque, pelas pressões das circunstâncias. Enquanto os enfermeiros mais experientes, a visão sobre a morte é mais apurada, pela vivência da mesma situação por dias diversos e com diferentes pacientes. Mas o tempo de atuação não exclui o profissional das dificuldades em lidar com a morte dos pacientes. ${ }^{18}$

Os comportamentos dos profissionais oscilam quando estão diante a morte, desde o abandono, com a intenção de preservação de sentimento de perda, e os que acabam por programar intervenções que trazem apenas o prolongamento do sofrimento do paciente e não promovem a morte com qualidade. ${ }^{11}$

É importante desenvolver uma abordagem integral e adequada diante da finitude da vida. A equipe de enfermagem precisa associar seus conhecimentos científicos ao objetivo de aliviar o sofrimento e dor do paciente, serem capacitados continuamente para lidar com a finitude da vida em qualquer setor do hospital, e com maior especificidade na pediatria. Sendo a equipe de enfermagem altamente relevante neste processo, pois através deles são disponibilizados os recursos para o cuidado, desde aquisição até a finalidade do uso das tecnologias da rede de assistência. ${ }^{19}$

$\mathrm{O}$ enfermeiro avalia a demanda de atividades e materiais, planeja as ações e auxilia na implementação das intervenções. E o técnico de enfermagem participa ativamente das intervenções a serem realizadas. ${ }^{19}$

A literatura científica registra a dificuldade do profissional de aceitar a morte infantil como algo natural. Em uma sociedade que nega a morte, faltam aos profissionais atributos psicológicos que os ajudem a acompanhar o estágio final de crianças e pacientes neonatais. ${ }^{20}$

Esse processo afeta significativamente a vida dos profissionais de enfermagem. A maneira como cada um deles busca compreender a morte e acompanhar o paciente, de acordo com suas experiências profissionais e crenças pessoais, faz diferença na forma de enfrentamento. Estudos demonstram que, com o passar do tempo, a rotina de viver experiências dolorosas gera mecanismos de defesa que levam os profissionais a tentar se manter indiferentes às circunstâncias que anteriormente os afligiram. Assim, tal como ocorre na sociedade, que tenta omitir a morte no cotidiano, a enfermagem reproduz esse mecanismo de defesa. ${ }^{20}$

São presentes diante a morte, comportamentos de insegurança e afastamento, causados por sentimentos de medo por relacionar a uma falha terapêutica, raiva por não conseguir alcançar a cura, sentir culpa e fracasso na assistência, estes sentimentos podem ocorrer separadamente ou uma junção de sentimentos e comportamentos negativos. Direcionando para a necessidade de ampliar os planejamentos de assistências, a discussão sobre os casos e a busca de aperfeiçoamento profissional. ${ }^{16,19}$

Os profissionais apontam a dificuldade de lidar com o paradoxo entre o sofrimento e o alívio com a morte vivenciada pelo indivíduo, e que a finalidade da vida na pediatria traz uma sobrecarga emocional. Também são explicados pelo fato de o relacionamento interpessoal do enfermeiro com uma criança internada, com o tempo vai criando laços afetivos, cada dia mais intensos, que a equipe de enfermagem inclui como parte da sua família. ${ }^{19}$

Estes são fatores que dificultam os profissionais da saúde de lidar com a morte das crianças nos setores de pediatria, e por este motivo, alguns, evitam o sofrimento e sentimento de perda, afastando-se dos pacientes e limitando suas habilidades a execução das suas competências técnicas. ${ }^{19}$

\section{Conclusão}

Conclui-se que o enfrentamento do processo morte e morrer emerge diversas complicações pra equipe de enfermagem e, quando ocorre na idade pediátrica, em situações de emergência é ainda mais desfavorável e, por isso, precisa ser tratado com muito cuidado e cautela.

Porsuavez, ressalta-sequea atividadenão somente da equipe de enfermagem, mas também de toda equipe multiprofissional, de oferecer suporte, informação, conforto e dignidade ao paciente e sua família por meio da assistência. São necessários estes suportes e subsídios, pois a criança vivencia sentimentos de medo, abandono, culpa e comportamentos depressivos.

Por fim, baseado nos achados literários desta construção, nota-se, ainda mais, que a vivência com a morte de seus pacientes pediátricos causa impactos negativos na equipe de enfermagem, mesmo após anos de atuação na área de emergência pediátrica, e que tem influência significativa em sua qualidade de vida que, se não discutida, pode interferir na assistência prestada a outros pacientes, tendo em vista o impacto causado na equipe. 


\section{Referências}

1. Matsumoto, DY. Cuidados paliativos: conceitos, fundamentos e princípios. Manual de cuidados paliativos ANCP, 2 (2):23-24, 2012.

2. Pessini, L; Hossne, WS. Terminalidade da vida e o novo Código de Ética Médica. Revista - Centro Universitário São Camilo, 4(2):27-129, 2010 .

3. Antunes, IO; Mota, ISS; Souza, AAM. Vivenciando a Morte na Pediatria: sofrimento da Equipe de Enfermagem. Revista Multidisciplinar das FIPMoc, (10):04-09, 2011.

4. Combinato, DS; Queiroz, MS. Um estudo sobre a morte: uma análise a partir do método explicativo de Vigotski. Ciência \& Saúde Coletiva, 16(9): 2011.

5. Avanci, BC. Cuidados paliativos à criança oncológica na situação do viver/morrer: a ótica do cuidar em enfermagem. Esc Anna Nery RevEnferm, 13(4):708- 16, 2009.

6. Oliveira, SG, Quintana, AM, Bertolino, KCO. Reflexões acerca da morte: um desafio para a enfermagem. Revista Brasileira de Enfermagem, 63(6), 2010.

7. Gil, AC. Como elaborar projetos de pesquisa. 5. ed. São Paulo: Atlas, 2008

8. Minayo, MCS. O desafio do conhecimento: pesquisa qualitativa em saúde. In: O desafio do conhecimento: pesquisa qualitativa em saúde. 2014

9. Lakatos, EM; Marconi, NA. Fundamentos de Metodologia Cientifica. 7. ed. São Paulo. Ed. Atlas, 2010.

10. Rodrigues, JC. A morte como um tabu. ComCiência, Campinas, n. 163, nov. 2014

11. Cegalla, DO. Dicionário escolar da língua portuguesa. 2 ed. São Paulo, Companhia Editora Nacional, 2008.

12. Osswald, W. Sobre a morte e o morrer. Fundação Francisco Manuel dos Santos, 2016

13. Marengo, MO; Flávio, DA.; Silva, RHA. Terminalidade de vida: bioética e humanização em saúde. Medicina (Ribeirão Preto. Online), 42 (3): 350-357, 2009

14. Santos, RA; Moreira, MCN. Resiliência e morte: o profissional de enfermagem frente ao cuidado de crianças e adolescentes no processo de finitude da vida. Ciência \& Saúde Coletiva, 19 (12): 4869-4878, 2014

15. Santos, EC. Validação de protocolo assistencial de enfermagem para o paciente em processo de terminalidade. [Dissertação] Universidade Federal do Rio Grande do Norte. Natal, 2015.

16. Parentoni, Camila da Costa. Atuação do enfermeiro diante da terminalidade e morte da criança e do adolescente com câncer em cuidados paliativos. [Dissertação]. Universidade de Estadual de Campinas. 2015.

17. Lima, MMSM. Prevalência de trauma facial em crianças e adolescentes vítimas de acidentes de trânsito. [Trabalho de Conclusão de Curso] Universidade Estadual de Paraíba. Campina Grande, 2014.

18. Faria, TNTet al. Cuidados paliativos em unidade de terapia intensiva: percepções dos profissionais de enfermagem. Rev. enferm. UFPE online, 11(5): 1996-2002, 2017.

19. Ribeiro Hermes, H; Arruda Lamarca,IC. Cuidados paliativos: uma abordagem a partir das categorias profissionais de saúde. Ciência \& Saúde Coletiva, 18(9), 2013 